\title{
Circular RNA circHIPK3 serves as a prognostic marker to promote chronic myeloid leukemia progression
}

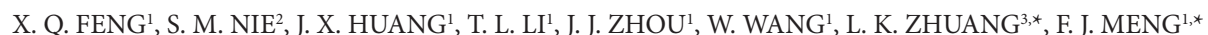

${ }^{1}$ Department of Hematology, The Affiliated Hospital of Qingdao University, Qingdao University, Qingdao University, Qingdao, China; ${ }^{2}$ Department of Neurology, The Affiliated Hospital of Qingdao University, Qingdao University, Qingdao, China; ${ }^{3}$ Institute of Transplantation Science, The Affiliated Hospital of Qingdao University, Qingdao University, Qingdao, China

*Correspondence: zlk0823@163.com, mengfanjunqdu@sohu.com

Received November 29, 2018 / Accepted June 4, 2019

\begin{abstract}
Increasing evidence demonstrate that circular RNAs (circRNAs) play critical role in regulation of gene expression, which participate in the pathogenesis of cancer, including chronic myeloid leukemia (CML). In this study, we aimed to investigate the expression profiling of circHIPK3 in CML. We found that circHIPK3 was significantly upregulated in peripheral blood mononuclear cells (PBMC) and serum samples from CML compared with healthy controls. High circHIPK3 expression predicted a poor outcome of CML patients. Further loss-function experiments suggested the oncogenic role of circHIPK3 in CML. Our findings provide insights on the role of circHIPK3 in the development and treatment of CML.
\end{abstract}

Key words: chronic myeloid leukemia, circRNAs, outcome, miR-124

In the past, circular RNAs (circRNAs) were initially considered as by-products of mis-spliced RNAs. Recently, by circRNA microarray, a large number of circRNAs have been identified. In addition, increasing evidence demonstrate that circRNAs play critical role in regulation of gene expression, which participate in the pathogenesis of cancer, including hematologic malignancies [1]. Several studies shown that circRNAs are abundantly expressed in the hematologic cancer cells and highly conserved across species and show tissue- and developmental stage-specific expression [2]. For example, circBA9.3 that derived from BCR-ABL1 can efficiently promote proliferation and inhibit apoptosis of cancer cells, and promote resistance against tyrosine kinase inhibitors therapy in chronic myelogenous leukemia [3]. hsa_circ_0080145 knockdown significantly suppressed chronic myeloid leukemia (CML) cell proliferation by acting as a miR-29b sponge [4].

CML is caused by a reciprocal translocation between chromosomes 9 and $22 \mathrm{t}(9 ; 22)$ (q34;q11), which account for up to $15 \%$ of reported cases of leukemia. The Ph chromosome is a reciprocal translocation, which results in a fusion gene and resultant transcription of the fusion protein BCR-ABL that has constitutive tyrosine kinase activity. Given the general deregulation in splicing mechanisms in CML, altered circRNAs could contribute to leukemogenesis. Previous study revealed that circHIPK3 is derived from Exon2 of the HIPK3 gene and directly binds to miR-124 and inhibits miR-124 activity [5]. The following studies demonstrate that circHIPK3 functions as biomarker for several cancers, such as glioma, ovarian cancer and osteosarcoma [6-8], and acts as an oncogene in lung cancer and colorectal cancer [9-10]. However, the role of circHIPK3 in chronic myeloid leukemia (CML) remains unknown.

In this study, we aimed to investigate the expression profiling of circHIPK3 in CML. We found that circHIPK3 was significantly upregulated in peripheral blood mononuclear cells (PBMC) and serum samples from CML compared with healthy controls. High circHIPK3 expression predicted a poor outcome of CML patients. Further loss-function experiments suggested the oncogenic role of circHIPK3 in CML. Our findings provide insights on the role of circHIPK3 in the development and treatment of CML.

\section{Patients and methods}

Patients and samples. One hundred patients with CML and age-matched healthy donors were enrolled in this study from The Affiliated Hospital of Qingdao University between Sep 2010 and Oct 2017. The medical records of CML patients with Sokal relative risk (RR) and survival information were 
collected. BCR/ABL fusion protein was detected by FISH in our hospital (Figure 1). Peripheral blood samples were collected from all subjects enrolled in the study. Peripheral blood mononuclear cells (PBMC) were obtained by FicollHypaque density gradient centrifugation and then stored at $-80^{\circ} \mathrm{C}$ in guanidinium thiocyanate until use. This project was approved by the Ethic Committee of The Affiliated Hospital of Qingdao University. Written informed consents were received from all subjects.

Cell culture and transfection. The human cell line 1D3 and 5 CML cell lines (K562, KCL22, AR230-r, LAMA84-s and Kasumi-4) were obtained from ATCC. Cells were grown routinely in RPMI-1640 medium (Invitrogen, CA, USA) supplemented with $10 \%$ fetal bovine serum (Gibco, CA, USA) and cultured in a $37^{\circ} \mathrm{C}$ humidified atmosphere of $5 \% \mathrm{CO}_{2}$. To test the effect of lentivirus circHIPK3 shRNA (circHIPK3 sh1, sequence: CCGGACTGTTGGCCAGTATGAATATCTCGAGATATTCATACTGGCCAACAGTTTTTTG; circHIPK3 sh2, sequence: CCGGTCTACATCAAATGGGTCTTTGCTCGAGCAAAGACCCATTTGATGTAGATTTTTG, Sigma-Aldrich), K562 cells were infected with either circHIPK3 sh1/sh2 (MOI = 100) or the empty lentivirus for $48 \mathrm{~h}$ in accordance with the manufacturer's instructions.

Quantitative real-time PCR analysis. Trizol reagent (Invitrogen) was used to extract total RNA from cells or serum samples. RevertAid First Strand cDNA Synthesis Kit (ThermoFisher Scientific, Waltham, MA, USA) was used to reverse transcript cDNA from total RNA according to the manufacturer's protocol. The expression of circRNAs was measured by PowerUp SYBR ${ }^{\mathrm{mm}}$ Green Master Mix (ThermoFisher Scientific) according to manufacturers' instructions. Expression of $\beta$-actin was used as an endogenous control. The primers were used as following: 5'-TGGAGACTGGGGGAAGATGA-3' (forward) and 5'-CACACTAACTGGCTGAGGGG-3' (reverse) for circHIPK3; 5'-GACCTGAGGAGATCAAGCCG-3' (forward) and 5'-ACTCCTCACTCTTGCGCTTC-3' (reverse) for HIPK3; 5'-TAGAACTGCACGGGAAACCC-3' (forward) and $5^{\prime}$-ACTATCCAGCACCTCCCACT-3' (reverse) for IGF2BP3; 5'-GTCTCTCCTCACAAGGTGGC-3' (forward) and 5'-ACTCCTCACTCTTGCGCTTC-3' (reverse) for B4GALT1;5'-CCTTCCAAGAAGAGCAGCGTG-3' (forward) and 5'-TGCCCAGAAGGAAACACCAT-3' (reverse) for p65; and 5'-TTGTTACAGGAAGTCCCTTGCC-3' (forward), 5'-ATGCTATCACCTCCCCTGTGTG-3' (reverse) for $\beta$-actin. All-in-One ${ }^{\mathrm{int}}$ miRNA qRT-PCR Reagent Kits (iGene Biotechnology Co., Ltd, Guangzhou, China) was used to measure miR-124 expression in serum samples, and U6 was used as an endogenous reference. $2^{-\Delta \Delta \mathrm{Ct}}$ method was used to calculate the relative expression.

Cell proliferation analysis. K562 and Kasumi 4 cells (1000 cells per well) were seeded into 96-well plates. After incubation at $37^{\circ} \mathrm{C}$ for $24 \mathrm{~h}, 48 \mathrm{~h}, 72 \mathrm{~h}$ and $96 \mathrm{~h}, 0.5 \%$ MTT solution was added to cells. After incubation at $37^{\circ} \mathrm{C}$ for $4 \mathrm{~h}, 150 \mu \mathrm{l}$ DMSO was added into each well to dissolve the formazan. The optical density $(570 \mathrm{~nm})$ was detected using a microplate reader (Bio-Rad Laboratories, CA, USA).

Cell apoptosis analysis. K562 and Kasumi4 cell apoptosis was analyzed by using Annexin V-FITC Apoptosis Staining/ Detection Kit (Abcam, Cambridge, MA, USA) following the manufacturer's instructions. Briefly, $2 \times 10^{5}$ cells were harvested by centrifugation at $1000 \mathrm{xg}$ for $5 \mathrm{~min}$ and resuspended in $100 \mu \mathrm{l}$ binding buffer, followed by a $15 \mathrm{~min}$ incubation with $5 \mu \mathrm{l}$ Annexin V-FITC in the dark at $37^{\circ} \mathrm{C}$. After that, $10 \mu \mathrm{l}$ PI staining was added with gentle shaking for 10 min incubation in the dark at $37^{\circ} \mathrm{C}$. Flow cytometry (BD) analysis was employed for detecting apoptotic events with FlowJo software (version 10, FlowJo, LLC, USA).

Western blot. Radioimmunoprecipitation assay (RIPA) lysis buffer (Boster, Wuhan, China) was used to extract protein from cells. BCA Protein assay kit (Thermo Scien-
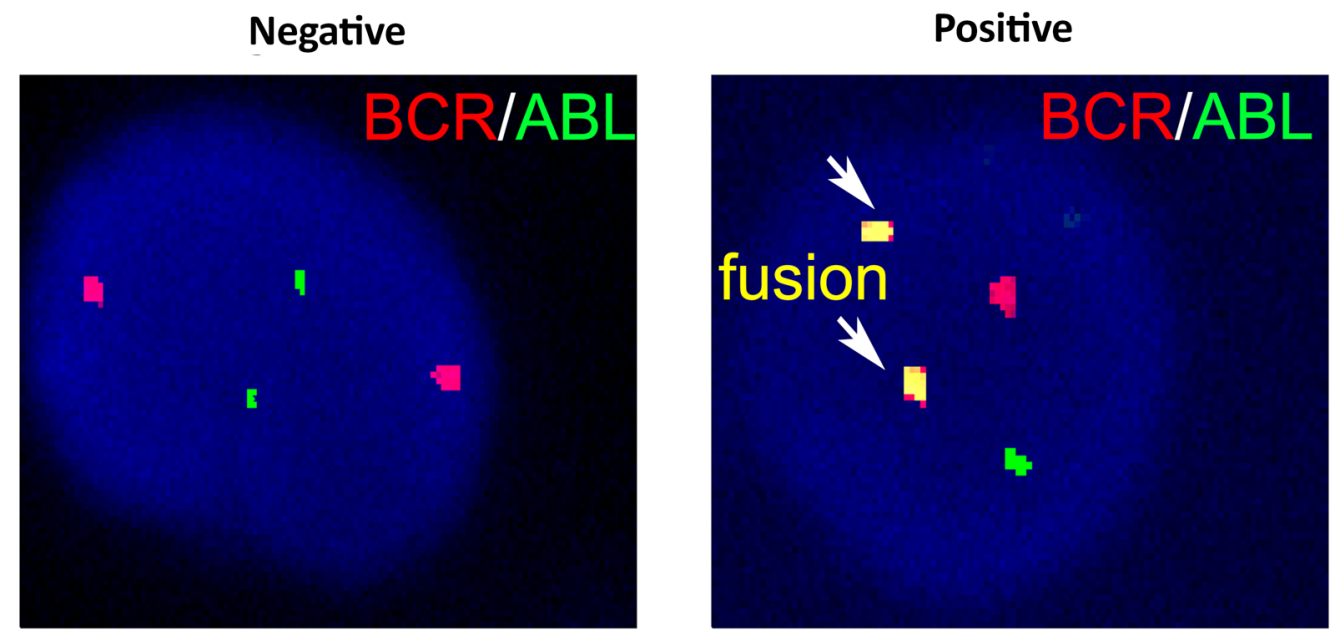

Figure 1. Representative BCR-ABL fusion positive and negative images by FISH. Red indicates BCR probes, blue indicates ABL probes and yellow indicates the BCR-ABL fusion protein. 
A

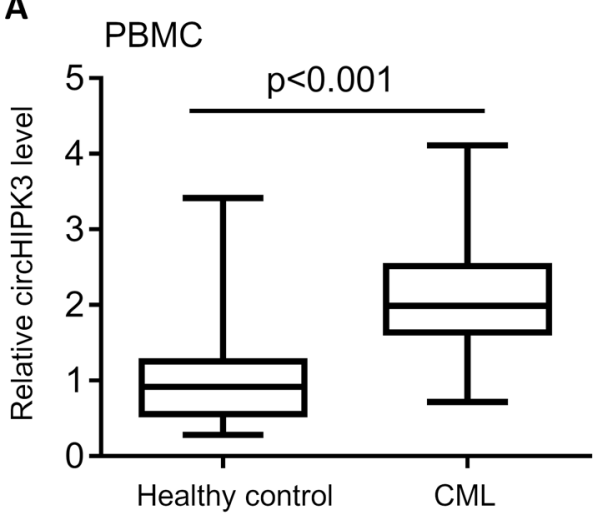

B

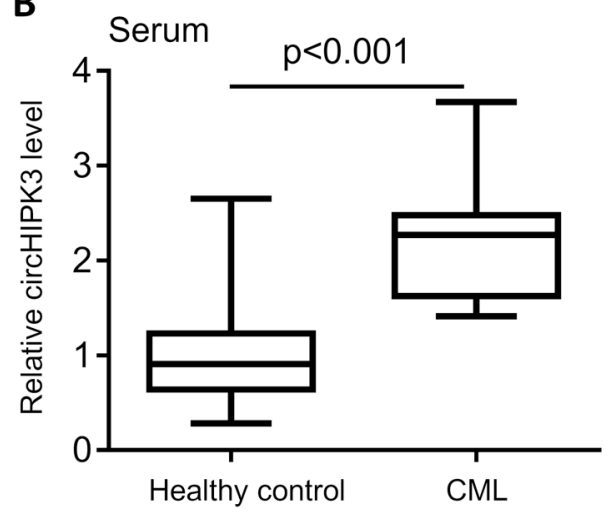

Figure 2. circHIPK3 expression in CML. A) qPCR was performed to measure the expression of circHIPK3 in PBMC from CML patients (n=100) and healthy controls $(n=100)$. B) qPCR was performed to measure the expression of circHIPK3 in serum samples from CML patients $(n=100)$ and healthy controls ( $\mathrm{n}=100)$. CML, chronic myeloid leukemia; PBMC, peripheral blood mononuclear cells.

tific) was used to measure protein concentrations. After separated by $10 \%$ SDS/PAGE, the proteins were blotted onto membranes. The membranes were then blocked by $5 \%$ non-fat milk for $30 \mathrm{~min}$ at room temperature and then immunoblotted with the following primary antibodies: $\mathrm{Bcl} 2$ antibody (cat no. 2872, diluted at 1:1000), Bax antibody (cat no. 5023, diluted at 1:1000), full length PARP antibody (cat no. 9532, diluted at 1:1000), cleaved PARP antibody (cat no. 5625 , diluted at 1:1000), caspase 3 antibody (cat no. 9662, diluted at 1:1000), cleaved caspase 3 antibody (cat no. 9664, diluted at 1:1000), GAPDH antibody (cat no. 5174, diluted at 1:3000). All antibodies were purchased from Cell Signaling Technology. Membranes were then incubated with peroxidase-conjugated secondary antibody and specific bands were detected with a Bio-Rad (Hercules, CA) imaging system.

Luciferase reporter gene assay. The 3'UTR of IGF2BP3, B4GALT1 and p65 were cloned downstream of FL reporter vector. Mutations were performed in the binding sites. 50 ng miR-124 mimics were obtained from ThermoFisher (Beijing, China). K562 cells were cultured for $24 \mathrm{~h}$ in 96 -well plates. Then, the cells were co-transfected with FL reporter, Renilla luciferase reporter and miRNA mimic for $48 \mathrm{~h}$. The luciferase activity was measured with a dual luciferase reporter assay system (Promega, Madison, WI) and normalized to Renilla activity.

Statistical analysis. All data from 3 independent experiments were expressed as mean \pm SD and processed using SPSS17.0 statistical software. The overall survival rate estimation was calculated using the Kaplan-Meier method with log-rank test. The clinical association between circHIPK3 expression and clinicopathological variables in CML patients was evaluated by chi-square test. The difference among the groups was estimated by Student's t-test or one-way ANOVA with post hoc Turkey test. A p-value $<0.05$ was statistically significant.

\section{Results}

CircHIPK3 is upregulated in CML patients. To investigate whether circHIPK3 could be used as biomarker for CML diagnosis, we performed $\mathrm{qPCR}$ to measure the expression of circHIPK3 in PBMC and serum samples from CML patients and healthy donors. The results revealed that circHIPK3 expression was significantly upregulated in CML patients PBMC compared with healthy controls (Figure 2A). In addition, we also found that circHIPK3 was significantly increased in serum samples from CML patients compared with healthy controls (Figure 2B).

High serum circHIPK3 is associated with poor outcome of CML patients. We analyzed the association between serum circHIPK3 and clinicopathological features of CML patients. The cases of CML patients were divided into high circHIPK3 expression with their expression more than the mean of circHIPK3 levels, otherwise into low circHIPK3 expression group. We found that circHIPK3 expression was associated with Sokal relative risk $(\mathrm{p}=0.017)$, but not $B C R / A B L$ mutant status $(p=0.891)$, age $(p=0.536)$ and gender $(p=0.151)$ (Table 1$)$. In addition, we investigated the factors that could predict the prognosis of CML patients by using the univariate and multivariate analyses. Univariate analysis indicated that the serum circHIPK3 level $(p=0.01)$, as well as Sokal relative risk $(\mathrm{p}=0.02)$ and $\mathrm{BCR} / \mathrm{ABL}$ mutant status $(\mathrm{p}=0.01)$ was significantly associated with patients' prognosis (Table 2). Multivariate analysis revealed that the serum circHIPK3 level $(p=0.02)$, Sokal relative risk $(\mathrm{p}=0.01)$ and BCR/ABL mutant status $(\mathrm{p}=0.02)$ were found to be independent factors for predicating the prognosis of CML patients (Table 3 ). We further analyzed the relationship between serum circHIPK3 levels and survival time of CML patients. The Kaplan-Meier survival cure showed that the patients with high circHIPK3 expression had shorter 
Table 1. Clinical association between serum circHIPK3 levels and clinicopathological variables of patients with CML

\begin{tabular}{|c|c|c|c|}
\hline \multirow[b]{2}{*}{ Variable } & \multicolumn{2}{|c|}{ serum circHIPK3 } & \multirow{2}{*}{$\begin{array}{l}\chi^{2} \text { test } \\
\text { p-value }\end{array}$} \\
\hline & $\begin{array}{c}\text { Low expression } \\
(\mathrm{n}=38)\end{array}$ & $\begin{array}{c}\text { High expression } \\
(\mathrm{n}=62)\end{array}$ & \\
\hline Age & & & 0.536 \\
\hline$<60$ & 20 & 37 & \\
\hline$\geq 60$ & 18 & 25 & \\
\hline Gender & & & 0.151 \\
\hline Male & 16 & 36 & \\
\hline Female & 22 & 26 & \\
\hline Sokal RR & & & 0.017 \\
\hline Low & 18 & 13 & \\
\hline Intermediate & 11 & 22 & \\
\hline High & 9 & 27 & \\
\hline $\mathrm{BCR} / \mathrm{ABL}$ & & & 0.891 \\
\hline Mutated & 34 & 56 & \\
\hline Unmutated & 4 & 6 & \\
\hline
\end{tabular}

Sokal RR, Sokal relative risk.

Table 2. Univariate analysis of prognostic factors of CML.

\begin{tabular}{lcc}
\hline Variable & Hazard ratio & p-value \\
\hline Age $(\geq 60$ vs. $<60)$ & 1.32 & 0.46 \\
Gender (Male vs Female) & 0.93 & 0.28 \\
Sokal RR (high vs. low/intermediate) & 2.54 & 0.02 \\
BCR/ABL (mutated vs. unmutated) & 3.32 & 0.01 \\
serum circHIPK3 levels (high vs. low) & 4.63 & 0.01 \\
\hline
\end{tabular}

Sokal RR, Sokal relative risk.

Table 3. Multivariate analysis of independent prognostic factors of CML.

\begin{tabular}{lcc}
\hline Variable & Hazard ratio & p-value \\
\hline Sokal RR (high vs. low/intermediate) & 5.72 & 0.02 \\
BCR/ABL (mutated vs. unmutated) & 4.15 & 0.01 \\
serum circHIPK3 levels & 4.82 & 0.02 \\
\hline
\end{tabular}

Sokal RR, Sokal relative risk.

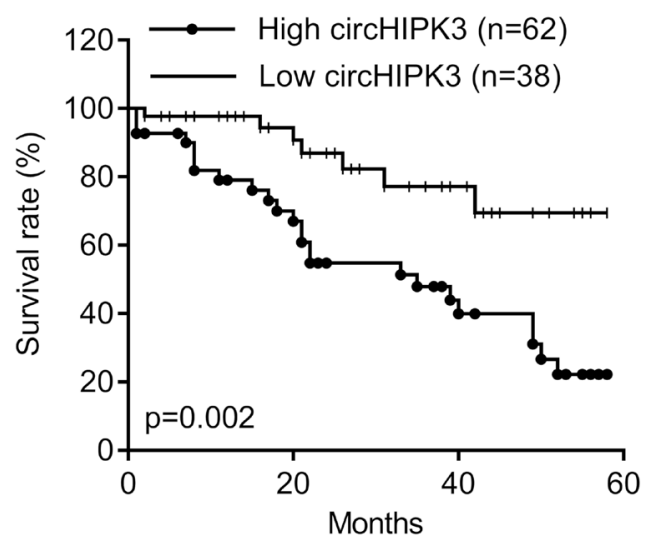

Figure 3. High circHIPK3 expression predicts a poor outcome for CML patients. The Kaplan-Meier survival cure showed the survival rate in patients with high circHIPK3 expression ( $n=62)$ and those with low circHIPK3 expression $(\mathbf{n}=38)$. overall survival rate than those with low circHIPK3 expression (Figure 3).

Knockdown of circHIPK3 by shRNA inhibits K562 and Kasumi4 cell growth. We next aimed to characterize circHIPK3 in CML cells. Compared with 1D3 cells, the expression of circHIPK3 was significantly upregulated in five CML cell lines, but the expression of host gene HIPK3 were comparable among these cell lines (Figure 4). Because of the highest amount of circHIPK3, we chose K562 and Kasumi4 cells for the next study. We first knocked down circHIPK3 expression in K562 and Kasumi4 cells (Figure $5 \mathrm{~A}$ ). The results showed that knockdown of circHIPK3 could significantly inhibit K562 and Kasumi4 cells proliferation (Figure 5B,C) and induced cell apoptosis (Figure 5D). In addition, our studies showed that downregulation of circHIPK 3 could lead to an apparent upregulation of bax, cleaved caspase 3 and cleaved PARP, and meanwhile the levels of bcl2 were substantially decreased, but the levels of full length PARP and pro-caspase 3 were comparable among groups (Figure 5E).

Given that circRNA has been shown to act as miRNA sponge and miR-124 was a valid target of circHIPK3, we therefore analyzed the expression of miR-124 and the correlation between miR-124 and circHIPK 3 in serum samples of CML patients. We found that the levels of miR-124 were significantly decreased in serum samples of CML patients compared with healthy controls (Figure 6A) and were negatively correlated with circHIPK3 expression (Figure $6 \mathrm{~B})$. Luciferase reporter gene assay and $\mathrm{qPCR}$ validated

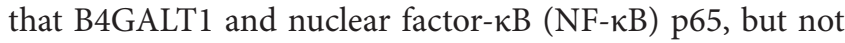
IGF2BP3, were the direct targets of miR-124 (Figures 6C, D), suggesting that circHIPK3/miR-124 axis may through these genes exhibits their functions.

\section{Discussion}

In this study, we found that circHIPK3 was significantly increased in CML PBMC and serum compared with healthy controls. High circHIPK3 expression predicted a poor outcome for CML patients. Further analysis showed that circHIPK3 would be an independent prognostic factor for CML patients' outcome.

Emerging evidence revealed the function of circRNAs in cancer and their potential to serve as a required novel biomarker and therapeutic target for cancer treatment. RNA sequencing revealed thousands of circRNAs in leukemia. For example, circPAN3 was increased in bone marrow samples from patients with refractory and recurrent acute myeloid leukemia. Downregulation of circPAN3 could decrease the expression of X-linked inhibitor of apoptosis protein, which was counteracted by miR-153-3p or miR-183-5p specific inhibitors [11]. circ-CBFB could serve as a diagnostic and prognostic biomarker for chronic lymphocytic leukemia patients. circ-CBFB knockdown significantly suppressed chronic lymphocytic leukemia cell proliferation, arrested 

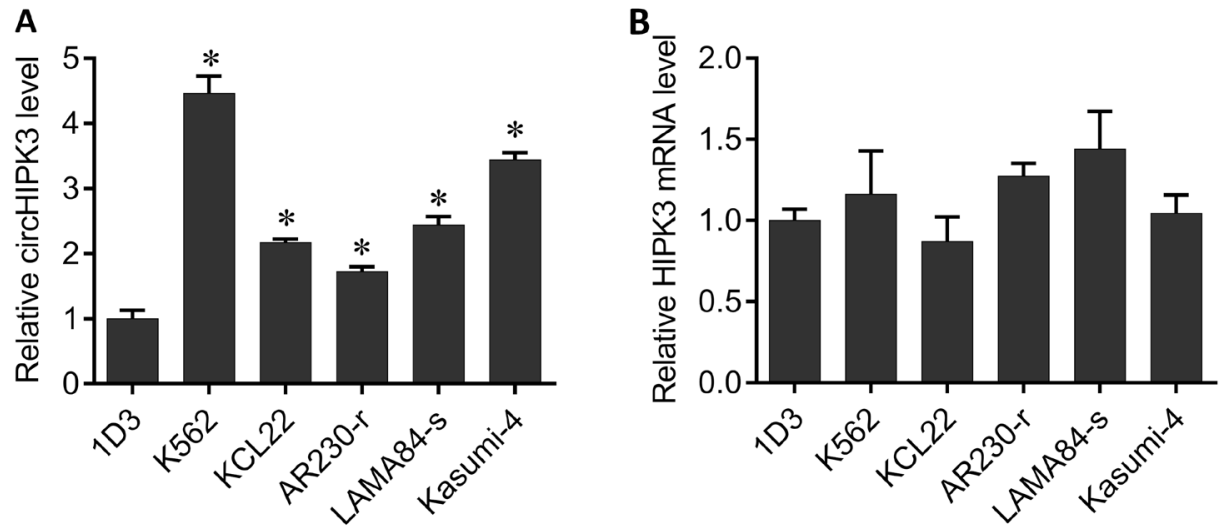

Figure 4. circHIPK3 expression in CML cell lines. qPCR was performed to measure the expression of circHIPK3 (A) and HIPK3 (B) in CML cell lines. ${ }^{*} \mathbf{p}<0.05$.
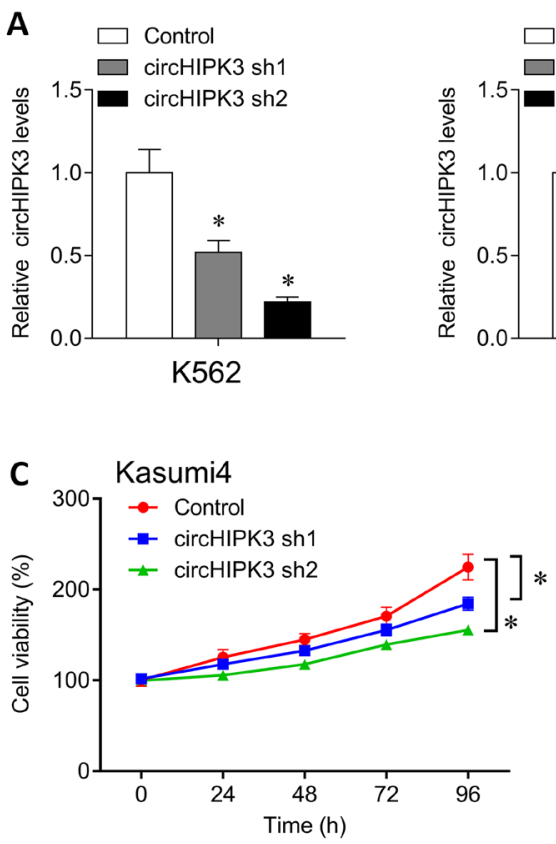
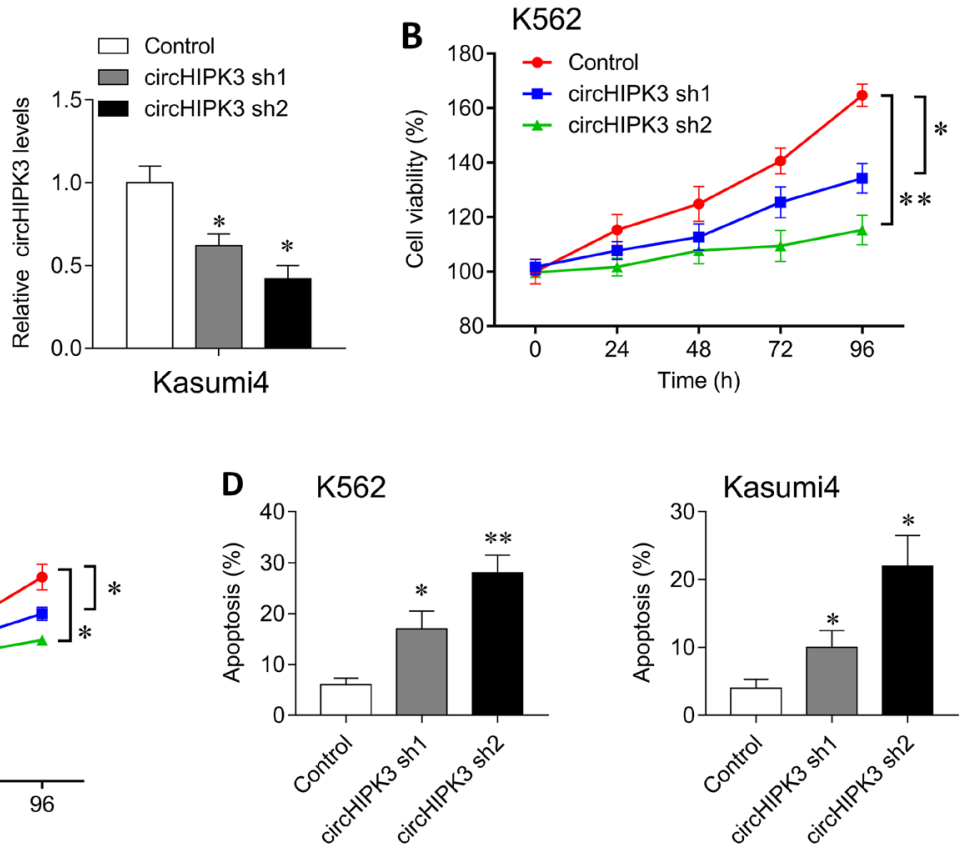
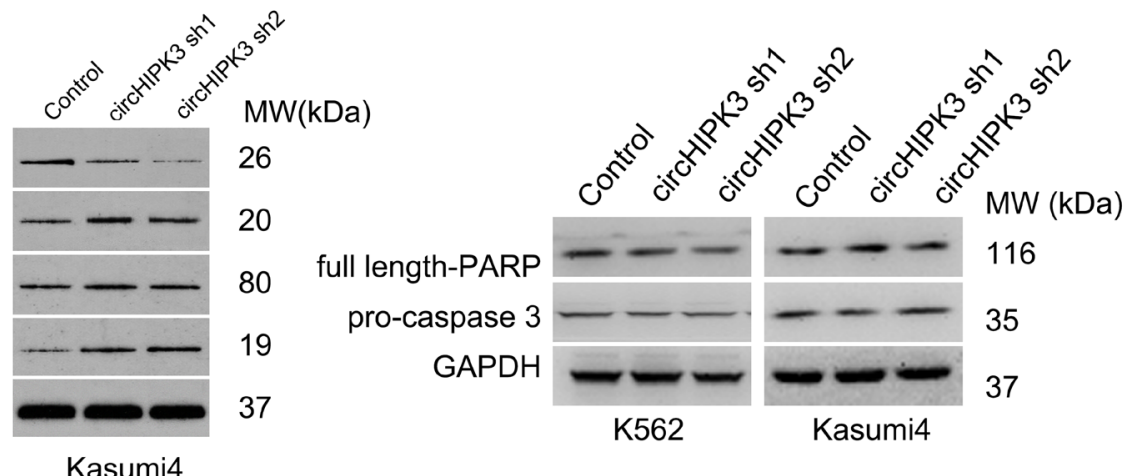

Figure 5. Knockdown of circHIPK3 inhibits K562 and Kasumi 4 cell proliferation. A) qPCR was performed to measure the expression of circHIPK3 after shRNA transfection in K562 and Kasumi4 cells. MTT was performed to evaluate the cell viability after shRNA transfection in K562 (B) and Kasumi4 (C) cells. D) Flow cytometry was performed to evaluate the cell apoptosis after shRNA transfection in K562 and Kasumi4 cells. E) Western blot was performed to evaluate the expression of apoptosis markers after shRNA transfection in $\mathrm{K} 562$ and Kasumi4 cells. ${ }^{\star} \mathrm{p}<0.05$, ${ }^{\star *} \mathrm{p}<0.01$. 
A

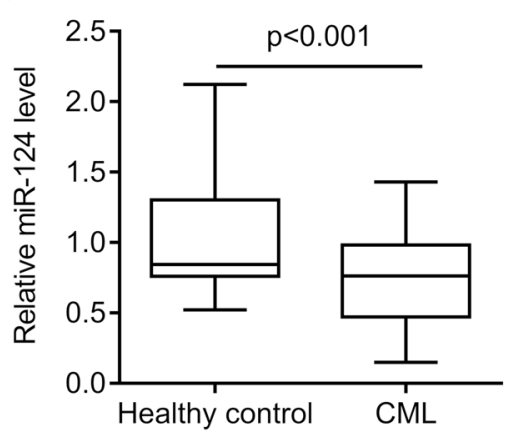

C

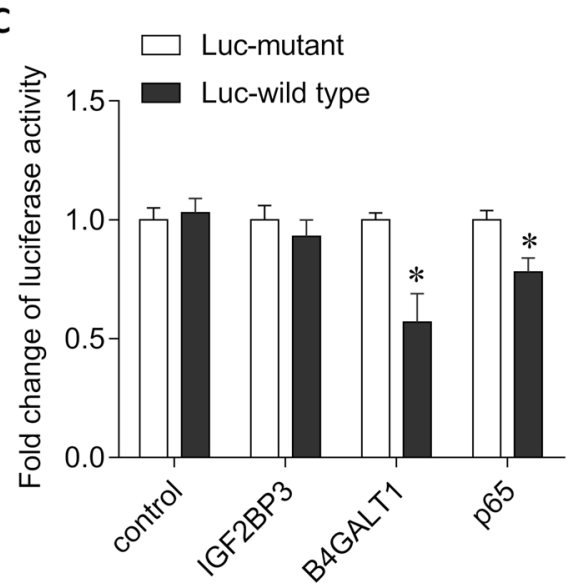

B
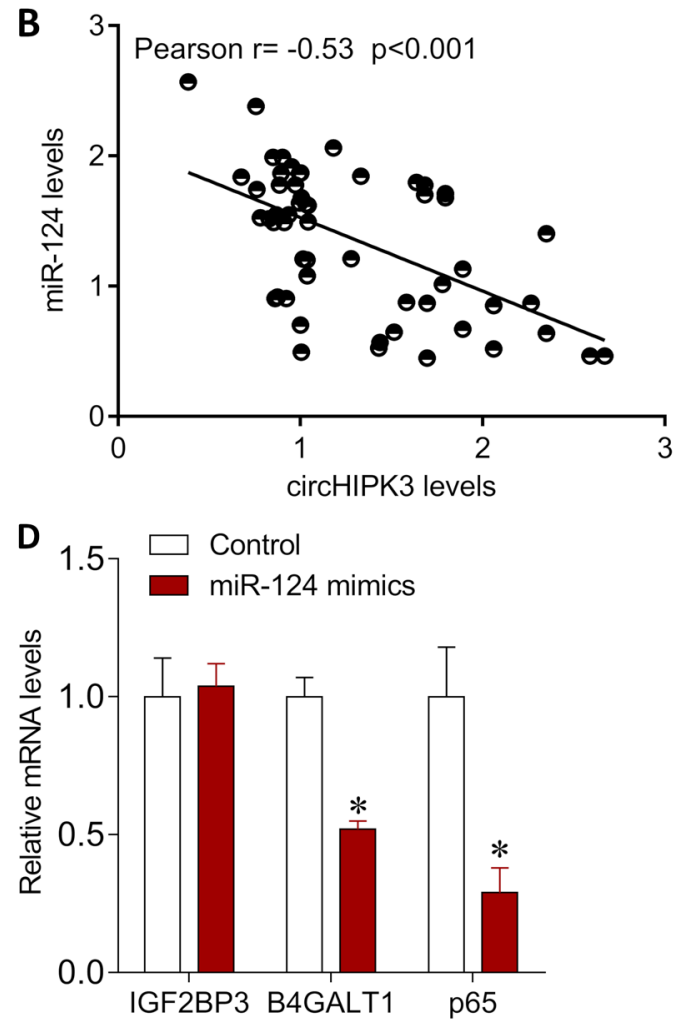

Figure 6. Association of miR-124 and circHIPK3. A) qPCR was performed to measure the expression of miR-124 in serum samples from CML patients $(n=100)$ and healthy controls $(n=100)$. B) miR-124 was negatively correlated with circHIPK3 in CML patients. C) Luciferase reporter gene assay was performed to analyze the targets of miR-124 in K562 cells. D) qPCR was performed to measure the expression of IGF2BP3, B4GALT1 and nuclear factor- $\kappa B(N F-\kappa B)$ p65 after miR-124 mimics transfection in K562 cells. ${ }^{*} \mathrm{p}<0.05$.

cell cycle progression, and induced cellular apoptosis by sponging miR-607, leading to the inactivation of the Wnt/ $\beta$-catenin pathway [12]. Here, we firstly revealed that high circHIPK3 was associated with poor outcome of CML patients and may be an independent prognostic factor for CML progression. Knockdown of circHIPK3 inhibited cell proliferation and induced cell apoptosis. Several studies have investigated the role of circHIPK3 on various types of cancer. However, it seems that the role of circHIPK3 is cancer-specific. CircHIPK3 is upregulated in lung cancer, nasopharyngeal carcinoma, glioma, epithelial ovarian cancer, hepatocellular carcinoma and colorectal cancer [6-7, 9-10, 13-14], but it is downregulated in bladder cancer, osteosarcoma [8, 15]. CircHIPK3 exhibited its oncogenic role by sponging miR-124, miR-654, miR-7 $[6,10,14]$, and acted as a tumor suppressor by binding to miR-558 [15]. MiR-124 has been demonstrated as tumor suppressor in many cancers, including CML, which is involved in p53 signaling and $\mathrm{NF}-\kappa \mathrm{B}$ pathway [16-18]. In recent study, we found that the expression of miR-124 was significantly decreased in serum samples from CML patients compared with healthy donors. Importantly, miR-124 levels were negatively correlated with circHIPK3 in CML patients. In addition, luciferase reporter gene assay and $\mathrm{qPCR}$ validated that B4GALT1 and nuclear factor- $\kappa \mathrm{B}(\mathrm{NF}-\kappa \mathrm{B})$ p 65 were the direct targets of miR-124. These genes have been reported as targets of miR-124 in glioma, B-cell lymphomas and CML $[16,18]$, suggesting that circHIPK3/miR-124 axis may through these genes to exhibit their functions.

In conclusion, our study firstly identified that circHIPK3 was significantly upregulated in CML PBMC and serum samples and might serve as a novel biomarker for CML. Our findings also provide a new insight of circHIPK3 on CML and a novel target for CML treatment.

\section{References}

[1] BONIZZATO A, GAFFO E, TE KRONNIE G, BORTOLUZZI S. CircRNAs in hematopoiesis and hematological malignancies. Blood Cancer J 2016; 6: e483. https://doi. org/10.1038/bcj.2016.81

[2] LUX S, BULLINGER L. Circular RNAs in Cancer. Adv Exp Med Biol 2018; 1087: 215-230. https://doi.org/10.1007/978981-13-1426-1_17 
[3] PAN Y, LOU J, WANG H, AN N, CHEN H et al. CircBA9.3 supports the survival of leukaemic cells by upregulating $\mathrm{C}-\mathrm{ABL} 1$ or BCR-ABL1 protein levels. Blood Cells Mol Dis 2018; 73: 38-44. https://doi.org/10.1016/j. bcmd.2018.09.002

[4] LIU J, KONG F, LOU S, YANG D, GU L. Global identification of circular RNAs in chronic myeloid leukemia reveals hsa_circ_0080145 regulates cell proliferation by sponging miR-29b. Biochem Biophys Res Commun 2018; 504: 660665. https://doi.org/10.1016/j.bbrc.2018.08.154

[5] ZHENG Q, BAO C, GUO W, LI S, CHEN J et al. Circular RNA profiling reveals an abundant circHIPK3 that regulates cell growth by sponging multiple miRNAs. Nat Commun 2016; 7: 11215. https://doi.org/10.1038/ncomms11215

[6] JIN P, HUANG Y, ZHU P, ZOU Y, SHAO T et al. CircRNA circHIPK3 serves as a prognostic marker to promote glioma progression by regulating miR-654/IGF2BP3 signaling. Biochem Biophys Res Commun 2018; 503: 1570-1574. https:// doi.org/10.1016/j.bbrc.2018.07.081

[7] LIU N, ZHANG J, ZHANG LY, WANG L. CircHIPK3 is upregulated and predicts a poor prognosis in epithelial ovarian cancer. Eur Rev Med Pharmacol Sci 2018; 22: 3713-3718. https://doi.org/10.26355/eurrev_201806_15250

[8] XIAO-LONG M, KUN-PENG Z, CHUN-LIN Z. Circular RNA circ_HIPK3 is down-regulated and suppresses cell proliferation, migration and invasion in osteosarcoma. J Cancer 2018; 9: 1856-1862. https://doi.org/10.7150/jca.24619

[9] YU H, CHEN Y, JIANG P. Circular RNA HIPK3 exerts oncogenic properties through suppression of miR-124 in lung cancer. Biochem Biophys Res Commun 2018; 506: 455-462. https://doi.org/10.1016/j.bbrc.2018.10.087

[10] ZENG K, CHEN X, XU M, LIU X, HU X et al. CircHIPK3 promotes colorectal cancer growth and metastasis by sponging miR-7. Cell Death Dis 2018; 9: 417. https://doi. org/10.1038/s41419-018-0454-8
[11] SHANG J, CHEN WM, WANG ZH, WEI TN, CHEN ZZ et al. CircPAN3 mediates drug resistance in acute myeloid leukemia through the miR-153-5p/miR-183-5p-XIAP axis. Exp Hematol 2019; 70: 42-54.e3. https://doi.org/10.1016/j. exphem.2018.10.011

[12] XIA L, WU L, BAO J, LI Q, CHEN X et al. Circular RNA circ-CBFB promotes proliferation and inhibits apoptosis in chronic lymphocytic leukemia through regulating miR-607/ FZD3/Wnt/beta-catenin pathway. Biochem Biophys Res Commun 2018; 503: 385-390. https://doi.org/10.1016/j. bbrc.2018.06.045

[13] KE Z, XIE F, ZHENG C, CHEN D. CircHIPK3 promotes proliferation and invasion in nasopharyngeal carcinoma by abrogating miR-4288-induced ELF3 inhibition. J Cell Physiol 2019; 234: 1699-1706. https://doi.org/10.1002/jcp.27041

[14] CHEN G, SHI Y, LIU M, SUN J. circHIPK3 regulates cell proliferation and migration by sponging miR-124 and regulating AQP3 expression in hepatocellular carcinoma. Cell Death Dis 2018; 9: 175. https://doi.org/10.1038/s41419-017-0204-3

[15] LI Y, ZHENG F, XIAO X, XIE F, TAO D et al. CircHIPK3 sponges miR-558 to suppress heparanase expression in bladder cancer cells. EMBO Rep 2017; 18: 1646-1659. https:// doi.org/10.15252/embr.201643581

[16] LIU YX, WANG L, LIU WJ, ZHANG HT, XUE JH et al. MiR124-3p/B4GALT1 axis plays an important role in SOCS3-regulated growth and chemo-sensitivity of CML. J Hematol Oncol 2016; 9: 69. https://doi.org/10.1186/s13045-016-0300-3

[17] WONG KY, SO CC, LOONG F, CHUNG LP, LAM WW et al. Epigenetic inactivation of the miR-124-1 in haematological malignancies. PLoS One 2011; 6: e19027. https://doi. org/10.1371/journal.pone.0019027

[18] JEONG D, KIM J, NAM J, SUN H, LEE YH et al. MicroRNA-124 links p53 to the NF-kappaB pathway in B-cell lymphomas. Leukemia 2015; 29: 1868-1874. https://doi. org/10.1038/leu.2015.101 